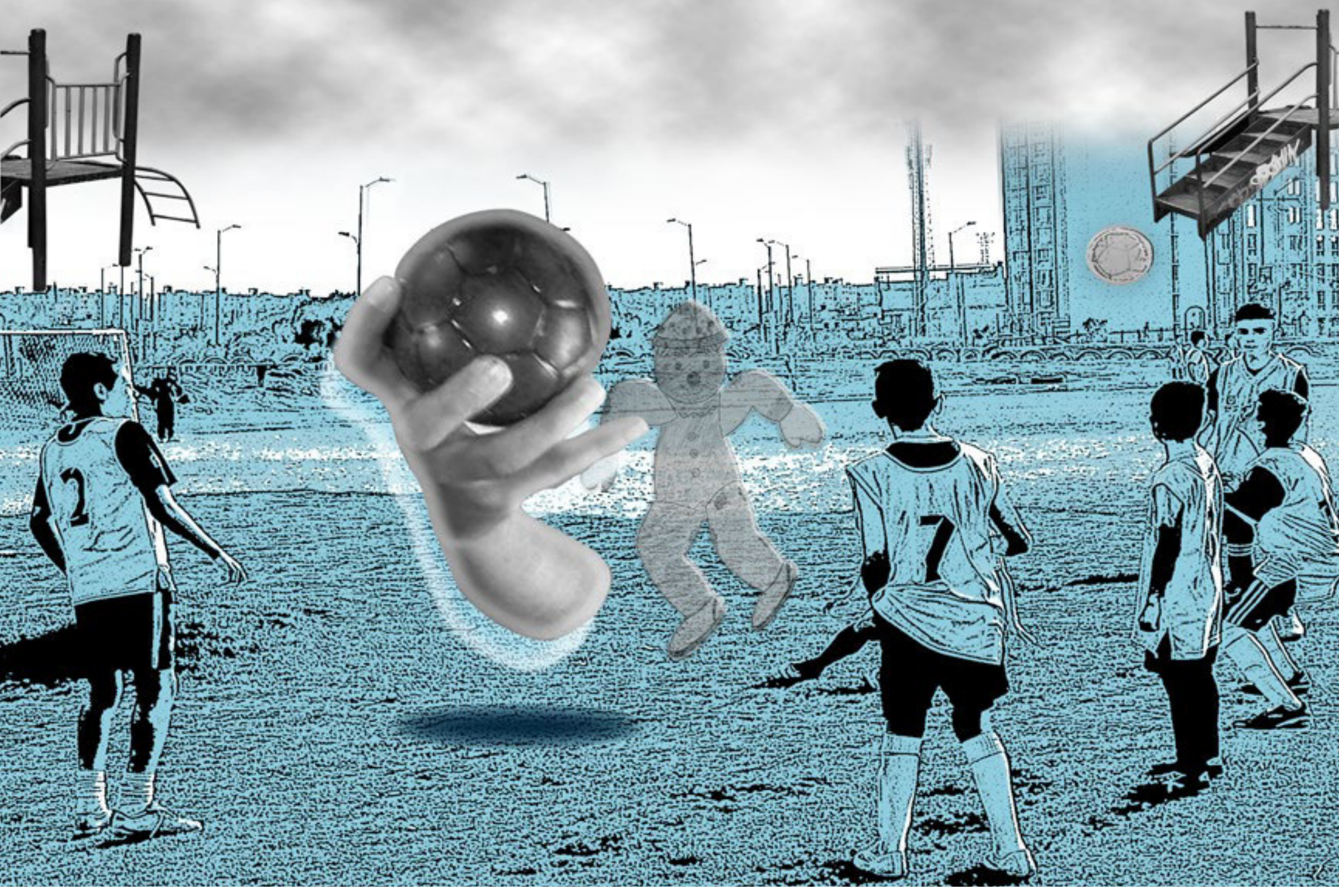

\title{
Prolegómenos para una "aprendencia" desde la corporeidad
}

Prolegomena for learning from the corporeality

Prolegômenos para um "aprendizado" da corporeidade Carlos Eduardo Pacheco Villegas 


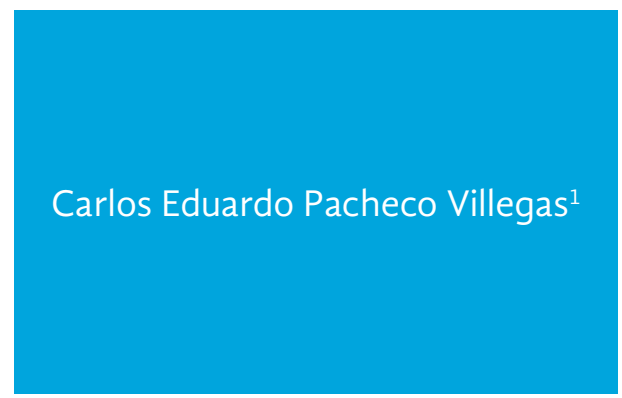

Candidato a Doctor en Filosofía, Universidad Santo Tomás. Magister en Educación, Universidad Pedagógica Nacional. Especialista en Gerencia Social de la Educación, UPN. Licenciado en Educación Física, UPN. Docente catedrático, Facultad Educación Física Universidad Pedagógica Nacional. Docente, SED Bogotá; correo electrónico: c_pachequin@yahoo.es

Fecha de recepción: 27 de noviembre de 2017 / Fecha de aprobación: 30 de marzo de 2018

\section{Resumen}

Como noción, la corporeidad está inmersa en lo que hoy se denominan "discursos epistemológicos emergentes de la Educación Física", con una fuerte carga fenomenológica inspirada en la intencionalidad, la conciencia y la experiencia humana. Su riqueza intrínseca invita a pensar desde "otras formas" la mediación pedagógica de la Educación Física, con la finalidad de superar la visión cartesiana dualista, cientificista y mecanicista que ha venido haciendo énfasis en la individualidad, la competitividad y el rendimiento. Esa visión cartesiana ha dejado de lado, aparentemente, la multidimensionalidad del sujeto aprendiente, la existencia humana y el ser. Se procura en el escrito, entonces, posibilitar la construcción de elementos formativos desde la corporeidad, que sugiera formas alternativas de aprendencia.

Palabras clave: Corporeidad, aprendencia, Educación Física.

\section{Abstract}

As a notion, corporeity is immersed in what are now called "emerging epistemological discourses of Physical Education", with a strong phenomenological burden inspired by intentionality, conscience and human experience. Its intrinsic wealth invites us to think from "other forms" the pedagogical mediation of Physical Education, in order to overcome the dualistic, scientistic and mechanistic Cartesian vision that has been emphasizing individuality, competitiveness and performance. This Cartesian vision has apparently left aside the multidimensionality of the learning subject, human existence and being. It is sought in the writing, then, to enable the construction of training elements from the corporeality, suggesting alternative forms of learning.

Keywords: Corporeity, learning, physical education.

\section{Resumo}

Como noção, a corporeidade está imersa no que hoje se denomina "discursos epistemológicos emergentes da Educação Física”, com forte carga fenomenológica inspirada na intencionalidade, consciência e experiência humana. Sua riqueza intrínseca nos convida a pensar, a partir de "outras formas”, a mediação pedagógica da Educação Física, a fim de superar a visão cartesiana dualista, cientificista e mecanicista que tem enfatizado a individualidade, a competitividade e o desempenho. Essa visão cartesiana aparentemente deixou de lado a multidimensionalidade do sujeito de aprendizagem, a existência humana e o ser. Procura-se, então, escrever para possibilitar a construção de elementos de treinamento a partir da corporeidade, sugerindo formas alternativas de aprendizagem.

Palavras-chave: Corporeidade, aprendizagem, Educação Física. 


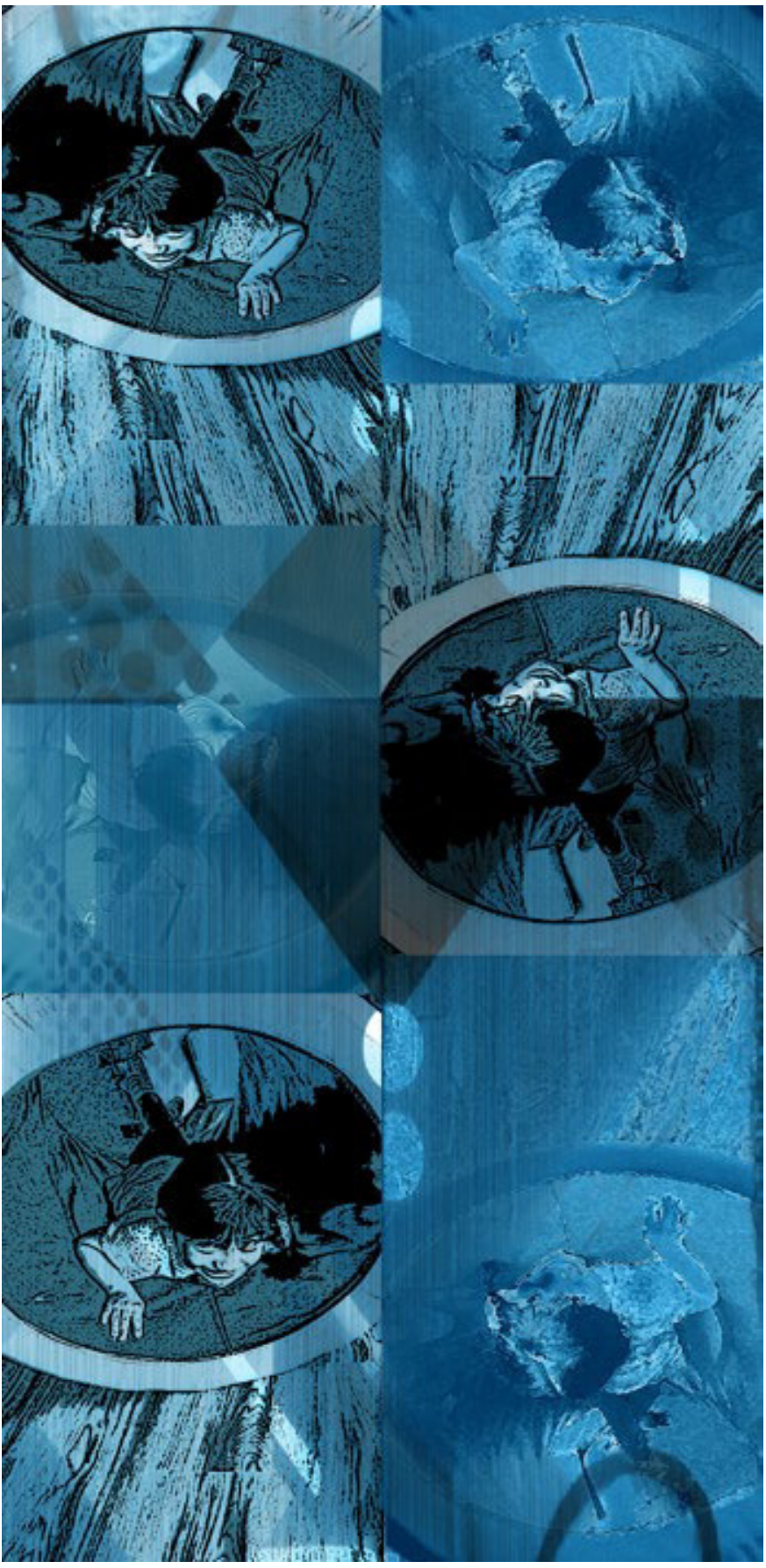

$\mathrm{E}$ n el campo de la Educación Física la colonialidad del saber se ha hecho evidente por las técnicas y metodologías aplicadas en su saber hacer, derivadas de las ciencias exactas que ponen el acento en el rendimiento y en el cuerpo como cosa. El ser humano en su integridad no ha sido prioridad para este enfoque cuantitativo de la disciplina y, en sí misma, la noción de Educación Física posee una fuerte carga semántica, deportivista y simbólica, que hace pensar que educar físicamente al hombre solo es posible si se siguen los postulados positivistas de las ciencias exactas.

Sin embargo, el horizonte que el desarrollo de la disciplina describe no es negativo ni pesimista, en la medida que se desea cuestionar y superar esas estructuras teóricas que han soportado las dinámicas formativas en la escuela. Los aportes teóricos de las universidades colombianas y los grupos de investigación, a propósito de la motricidad humana, la experiencia corporal y el cuerpo vivido, desde un enfoque fenomenológico ${ }^{2}$, han sugerido pensar la Educación Física de manera alternativa, tomando distancia del predominio de los datos y la lógica instrumental.

El escrito se desarrolla en dos momentos, el primero se relaciona con el panorama que describe el desbordamiento de la racionalidad científica, cristalizado en una crisis de las ciencias de la cual la Educación Física es heredera; y el segundo es un ejercicio de construcción teórica que propone la base de una aprendencia de la corporeidad a manera de prolegómenos. El intento se inscribe en los esfuerzos epistemológicos de formar al ser humano desde enfoques alternativos al tradicional.

\section{Primer momento}

Husserl hace uso de una categoría que caracteriza los desbordes racionales de la ciencia y amenaza al escepticismo, al irracionalismo y al misticismo, denominada "crisis". La caracterización de esos desbordes, planteados en este apartado, tiene que ver con una teorética y metódica sistemática justificada en la matemática pura

\footnotetext{
2. Son propuestas de un inquieto grupo de maestros en Colombia y en Latinoamérica: Margarita Benjumea, sobre los elementos constitutivos de la motricidad humana; el grupo de investigación Kon-traste, que adoptó lo sugerido por Manuel Sergio a propósito de la Ciencia de la motricidad humana; las aproximaciones epistemológicas de la profesora Luz elena Gallo en la Universidad de Antioquia; y León Urrego y William Moreno, cuyo objeto de estudio en la Licenciatura de Educación Física de la Universidad Pedagógica Nacional, se refirió a la experiencia corporal.
} 
y en un principio de exactitud (Husserl, 2008, p. 48). La "crisis" es, en primer lugar, un cuestionamiento de los métodos y las tareas que desarrolla y emplea la ciencia con la intención de universalizar las prácticas que ofrecen acceso al conocimiento; sobre todo una ciencia que se deriva del positivismo, del carácter científico y, como se ha mencionado, de la exactitud.

En segundo lugar, la "crisis" redefine un nuevo modelo de valoración de la ciencia, que no tenía en sus estimaciones a la existencia humana ni a los modos otros de ser del sujeto; es decir, esta nueva visión, en la segunda mitad del siglo XIX, deja de lado la subjetividad junto a todas aquellas preguntas que "son decisivas para una autentica humanidad" (Husserl, 2008, p. 50).

Con ello se podría decir, entonces, que "la crisis de las ciencias" es la denominación dada por Husserl a una forma de legitimación que aleja la existencia de la humanidad (su sentido y significado ubicado en la cultura) de una lógica formal y racional, porque la ciencia, con su lógica formal y racional, tecnifica, reduce y descarta preguntas relacionadas con lo enigmático de la consciencia, la sinrazón o la misma existencia. Esta ciencia se enfoca en la caracterización y objetividad de cuerpos inertes, de la repetición de unas constantes, de la aparición de fenómenos medibles y cuantificables. Sin embargo, no siempre las preguntas que se enfocaron en lo "humano" o, mejor, en la existencia humana, fueron desarraigadas de las ciencias en general.

En todos estos aspectos confluyeron diversas formas de plasmar la denuncia a ese reduccionismo y tecnicismo del conocimiento del hombre, que involucró categorías de análisis como ciencia, ciencia del hombre, ciencia natural, lógica, teorética, teoría de la ciencia, entre otras, como expresiones de una maraña lógica que sirvió de medio ambiente a las estructuras de pensamiento. Dentro de esa maraña se planteó precisamente el cuestionamiento de las ciencias alrededor de una tensión que desembocó en una inestabilidad a los fundamentos de la razón y a los fines que la ayudaron a promulgar.

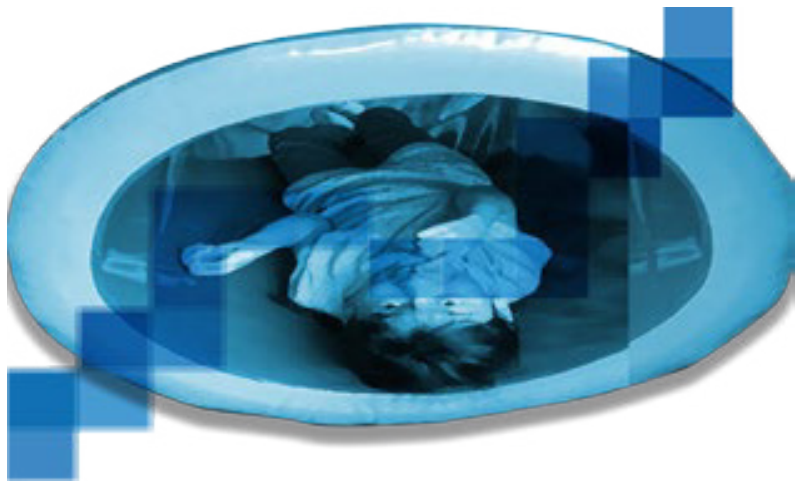

A finales del siglo XIX y comienzos del XX, las ciencias en general, pero en especial las denominadas Naturales, empezaron a ser cuestionadas por su carácter cientifícista y la manera institucionalizada de legitimar sus alcances. Durante años su método, validado y consolidado desde la institucionalidad científica, hizo ver su proceder como única fuente de acceso al conocimiento, un conocimiento legitimado por la creciente forma de ver la ciencia y la lógica desde la formalidad sin precedentes.

Cuando aflora la modernidad en el continente europeo, allí se imprime, instala y obliga a ver el mundo desde la lupa de un "No Dios", y del "No misterio" que caracterizaba la Edad Media, ofreciendo así una supuesta independencia arrogante al momento de dar una explicación a los problemas o fenómenos encontrados bajo el rótulo de "verdadero" o "auténtico". Es decir, eso "verdadero" o "auténtico" del carácter científico tuvo que ver con las nuevas apuestas relacionadas con los valores deterministas que orientaron su forma de ser y hacer, legitimados en la modernidad. En otras palabras, lo "autentico" se inscribió en una forma lógica y en una lógica interna que responde al espíritu propio de lo que la ciencia va construyendo y fortificando para ella misma. Los valores deterministas aquí son las imposiciones que se deben cumplir al pie de la letra, como mecanismo procedimental a seguir en la emergente doctrina científica.

Esa doctrina científica exigió toda forma de abandono a preguntas que la filosofía había tomado como relevantes para la existencia humana; como, por ejemplo: ¿Quién soy yo?; ¿ icuál es el sentido de la vida?; ¿qué es la libertad? y otras de esta índole, enmarcadas en un sentido metafísico, dejaron de ser atractivas para la ciencia, pues no podía tener un dominio real y universal sobre ellas. Es decir que, para el pensamiento científico de la época -comienzos de 1900-, las preguntas metafísicas sobrepasaban el mundo como universo de las mismas cosas y no fueron factibles para la comprobación desde las disciplinas científicas. Como se ha mencionado, el carácter científico descarta las preguntas que toman posiciones valorativas y relaciones derivadas de la cuestionabilidad de la razón y la sinrazón de la humanidad (Husserl, 2008, p. 50).

Este es el análisis relacional planteado por Husserl a la luz de un fracaso de la ciencia objetiva, al no interesarse por cuestiones propias de la humanidad y de no vislumbrar un panorama que produjera un conocimiento propio del hombre desde todas sus dimensiones antropológicas, sociológicas, metafísicas, espirituales, etc., conduciendo a una ciencia de hechos que estudiara meros hombres de hechos.

Con todo lo anterior, la manera en que la ciencia planteó su tarea se dio cerradamente en los bordes teóricos y producciones 
investigativas de lo que está hecho el mundo, pero le costó, y no hizo manifestaciones científicas de corte subjetivo; de hecho, el método científico tiene su mayor fecundidad solo en los albores de las disciplinas positivistas (Husserl, 2008, p. 54). En consecuencia, la ciencia prefiere alejarse de discusiones basadas en la espiritualidad, la subjetividad y la ambigüedad que sugiere descifrarse como humanidad de los hombres humanos, con la mera biologización de su corporalidad. En esa ambigüedad suelen emerger enigmas que solo pueden ser tratados y analizados desde la reflexión de las llamadas Ciencias Humanas, y precisamente es allí, dentro de esos estudios, que emergen otras formas de ser y de formar al ser.

\section{Segundo momento}

Bajo la racionalidad de la ciencia se originaron los sistemas de organización y producción en el mundo, anclando enunciados como sociedad del conocimiento, sociedad del aprendizaje, sociedad de consumo o aprendizaje por competencias, que pusieron en desventaja al ser humano, pues le introdujeron en una lógica de competición con los demás, aislándolo de su ser con una fuerte carga individualista, egoísta y hedonista.

Frente a ello, Assmann (2002) propone pensar una sociedad aprendiente, constituida por una actitud o un "estado de aprendizaje y transformarse en una inmensa red de ecologías cognitivas" (p. 19), es decir que la aprendencia, se diría en este escrito, es un estado alertado, en constante apertura con la disposición de aprender algo relacionado con el-mundo-de-la-vida. Para tal disposición no es prudente inclinar el enfoque hacia las formas de enseñar o a la enseñanza, sino que la mirada debe hacerse desde las posibilidades de "dejar ser"; en ese "dejar ser" confluyen diversos modos de ser del ser, que acogen una estructura ontológica en donde el Ser-ahí o la presencialidad -o como se conoce en la filosofía Dasein- es fundamental en la disposición de aprendencia desde la corporeidad.

En muchas ocasiones Husserl menciona el término "mundo de la vida" para designar algo que le es concreto y originario al hombre, resultado de la cultura en un momento histórico, sus problemas, sus soluciones y resoluciones teóricas planteadas desde una coherencia y acceso al mundo. La fenomenología va en busca de un retorno al mundo de la vida que está oscurecido y sombreado por diversas ideas, teorías, prejuicios, creencias que se han tejido en nuestro tiempo.

El mundo de la vida persigue todos los mundos de los hombres, sus metas, sus deseos, sus anhelos, que poseen una temporalidad

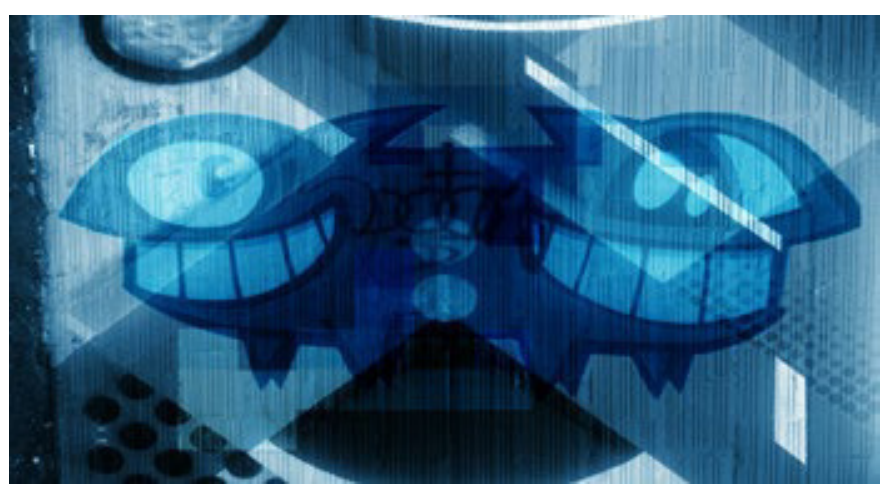

vivida que es muy diferente del tiempo homogéneo y medible, cuya estructura se enmarca en lo presente viviente, dimensión que es un privilegio del tiempo vivido. En este presente viviente se instalan las impresiones de las cosas presentes, la retención de nuestras experiencias pasadas y la protención de las experiencias futuras (Husserl, 2008, p. 116).

El mundo de la vida tiene en su estructura una dimensión temporal, pero también una dimensión espacial que orienta a nuestro propio cuerpo. Es decir, las dos dimensiones establecen un marco propio de elementos para la vida. El "olvido del ser" es, de forma rigurosa, el dejar de preguntar por aquello que se denomina "ser" y que fue parte esencial en las investigaciones de los antiguos griegos ${ }^{3}$. El olvido por la pregunta del ser posibilitó que la humanidad cayera en desgracia, por no reconocer, o no querer reconocer, la existencia humana como parte de una ontología cargada de sentido. "El ser es el concepto más universal, pero al mismo tiempo es algo indefinible porque no se pude definir como ente, y a la vez es evidente por sí mismo" (Parafraseando a Heidegger, 2006b, p. 27).

La esencia del ente del ser, dice Heidegger, consiste "en su tenerque-ser" (Zu-sein), el "Qué" (essentia) de este ente, en la medida en que se puede siquiera hablar así, debe concebirse desde su ser (existentia) (Heidegger: 2006b, §9, p. 67). Dentro del análisis existencial se contempla la pregunta por el ser y, en consecuencia, es el Dasein la fundamentación que abre la relación óntico-ontología.

En otras palabras, el Dasein heideggeriano obliga a pensar y a relacionar el análisis existencial del Dasein con el análisis de lo que significa ser-hombre. Dice Heidegger que: "el Dasein no es tan solo un ente que se presenta entre otros entes. Lo que lo caracteriza ónticamente es que a este ente le va en su ser este mismo ser". La constitución del ser del Dasein implica, entonces, que el Dasein

Para Heidegger, Platón y Aristóteles (Husserl, 2008, p. 26). 
tiene en su ser una relación de ser con su ser. Esto sería realmente lo novedoso de Heidegger: el hecho de identificar la caracterización de la "esencia" a diferencia de la caracterización de la "existencia", haciendo ver que el ser del ente es mío, lo cual significa, a su vez, que el Dasein se comprende en su ser de alguna manera y con algún grado de explicitud. Es propio de este ente el que con, y por su ser, se encuentre abierto y, sobre todo dispuesto, para él mismo. La comprensión del ser es ella misma una determinación de ser del Dasein. La peculiaridad óntica del Dasein consiste en que el Dasein es ontológico.

\section{Tercer momento}

La corporeidad, según Alicia Grasso (2001), es una: “construcción permanente de la unidad psicofísica-espiritual-motora-afectivasocial-intelectual"; es decir, "del ser humano a parte de lo que tiene significado para él y para su sociedad; es una concepción que rompe el habitus corporal que tiene incorporado el profesor, que busca la identidad corporal del alumno de educación física" (p. 19).

Parte de los prolegómenos para una aprendencia desde la corporeidad se fundamenta en el postulado de Assmann (2002), quien sostiene que: "solo una teoría de la corporeidad puede proporcionar las bases para una teoría pedagógica" (p. 32). Para la corporeidad, se consideran elementos constitutivos: a) La experiencia humana; b) La finitud motrícea; c) El espacio y el tiempo, y d) La Illeidad.

a. La experiencia humana. Según Mèlich (2012), se trata de "una pasión, un acontecimiento. Improgramable, implanificable, impensable [...] la experiencia es lo que nos sorprende, lo que nos rompe" (p. 67). En otras palabras, la experiencia humana pensar en esa actitud de alerta y dispuesta a aprender, desde

está llena de sentido, en la medida en que no es un recetario de cómo resolver los problemas y, solo por eso, ya tiene una carga de transformación hacia lo que desea o desconoce el aprendiente. La experiencia está anclada a un tiempo y a un espacio determinado; posibilita poder ser algo a alguien, pues no somos seres acabados. En cosecuencia, desde este elemento se puede captar el devenir de la existencia y dirigirlo a la formación del ser aprendiente.

b. La finitud motrícea. Es el elemento que posibilita identificar los alcances y límites de la dimensión motriz, relacionados con el lenguaje y la expresividad de mis pasiones. Aquí la experiencia humana despliega siempre un renovado lienzo en donde se impregnan las posibilidades de ser de otro modo.

c. El espacio y el tiempo. Se enmarcan en la determinación del recuerdo, ya que desde allí me permite volver sobre los rasgos que posibilitaron un acontecimiento, una emergencia o un padecimiento. El espacio se nutre de la heterotopia de sus lugares; se mimetiza como la geolocalización en el sueño: por instantes, colores, referencias cercanas y lejanas; aquí está el devenir

d. La Illeidad. Es un neologismo utilizado por Levinas (1995) e indica: "un modo de concernirme sin entrar en conjunción conmigo [...] la illeidad de lo-más-allá-del-ser es el hecho de que su venida hacia mí es un punto de partida que me permite realizar un movimiento hacia el prójimo" (p. 57). La illeidad en la aprendencia es la apertura, no solo hacia a mí, sino hacia la corporeidad del otro. No aprendemos solos, aprendemos junto al otro.

Estos cuatro elementos posibilitan pensar en una aprendencia desde la corporeidad, superando la carga epistemológica y de contenido que encierra la noción de Educación Física.
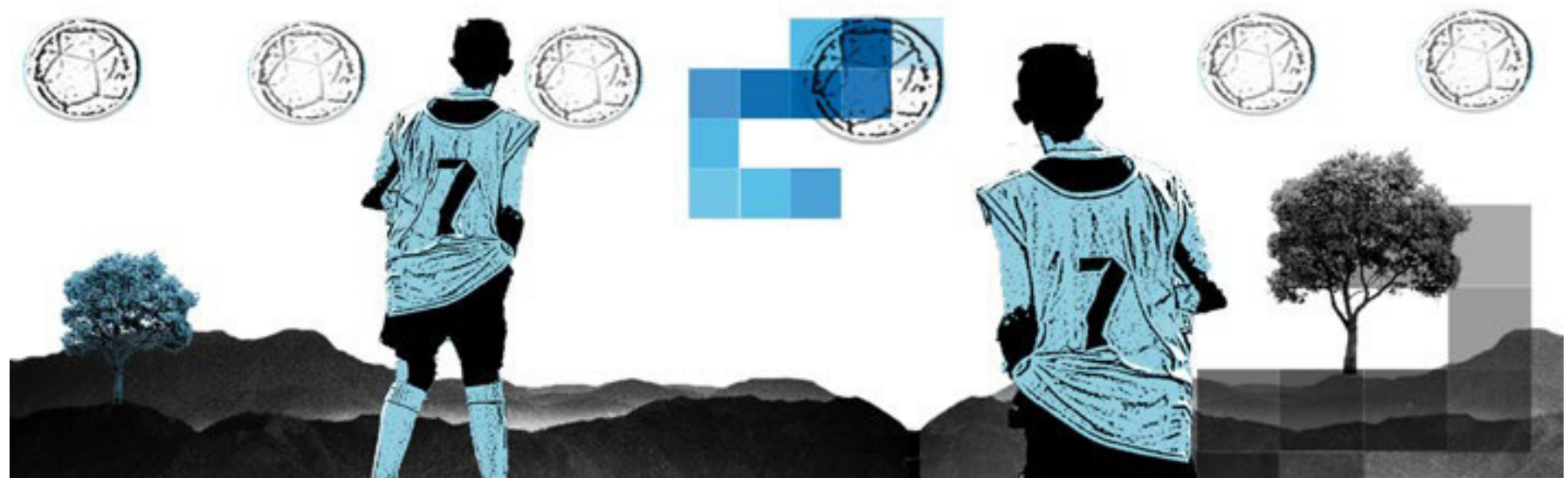


\section{Referencias}

Assmann, H. (2002). Placer y ternura en la educación. Hacia una sociedad aprendiente. Madrid: Narcea.

Grasso, A. (2001). El aprendizaje no resuelto de la educación física: la corporeidad. Buenos Aires: Ediciones Novedades Educativas.

Heidegger, M. (2006a). Carta sobre el Humanismo. Madrid: Alianza Editorial.

Heidegger, M. (2006b). Ser y tiempo. Madrid: Editorial Trotta.

Husserl, E. (2008). La crisis de las ciencias europeas y la fenomenología trascendental. Buenos Aires: Prometeo Libros.

Levinas, E. (1995). De otro modo que ser, o más allá de la esencia. Salamanca: Ediciones Sígueme.

Mèlich, J. (2012). Filosofía de la finitud. Barcelona: Herder. 